\title{
UNBOUNDEDNESS OF THE BALL BILINEAR MULTIPLIER OPERATOR
}

\author{
GEOFF DIESTEL AND LOUKAS GRAFAKOS
}

\begin{abstract}
For all $n>1$, the characteristic function of the unit ball in $\mathbb{R}^{2 n}$ is not the symbol of a bounded bilinear multiplier operator from $L^{p}\left(\mathbb{R}^{n}\right) \times L^{q}\left(\mathbb{R}^{n}\right)$ to $L^{r}\left(\mathbb{R}^{n}\right)$ when $1 / p+1 / q=1 / r$ and exactly one of $p$, $q$, or $r^{\prime}=r /(r-1)$ is less than 2 .
\end{abstract}

\section{$\S 1$. Introduction}

We denote the Fourier transform of a function $f$ on $\mathbb{R}^{n}$ by $\widehat{f}(\xi)=$ $\int_{\mathbb{R}^{n}} f(t) e^{-2 \pi i t \cdot \xi} d t$ and its inverse Fourier transform by $f^{\vee}(\xi)=\widehat{f}(-\xi)$. Let $B$ be the unit ball in $\mathbb{R}^{n}$ and $\chi_{A}$ the characteristic function of a set $A$. The unboundedness of the linear operator

$$
T_{\chi_{B}}(f)=\left(\widehat{f} \chi_{B}\right)^{\vee}
$$

on $L^{p}\left(\mathbb{R}^{n}\right)$ when $p \neq 2$ and $n>1$ was established by Fefferman [2].

In this article we provide a variant of Fefferman's result in the bilinear setting. Our arguments also work for multilinear operators. Let $1 \leq p_{1}, \ldots, p_{k} \leq \infty$ and $0<p<\infty$. We recall that a bounded function $m:\left(\mathbb{R}^{n}\right)^{k} \mapsto \mathbb{C}$ is called a $k$-linear multiplier if the $k$-linear operator

$$
\begin{aligned}
\left(f_{1}, \ldots, f_{k}\right) \longrightarrow \int_{\mathbb{R}^{n}} \ldots \int_{\mathbb{R}^{n}} m\left(\xi_{1}, \ldots, \xi_{k}\right) \widehat{f}_{1}\left(\xi_{1}\right) & \cdots \widehat{f}_{k}\left(\xi_{k}\right) \\
& \times e^{2 \pi i\left(\xi_{1}+\cdots+\xi_{k}\right) \cdot x} d \xi_{1} \cdots d \xi_{k}
\end{aligned}
$$

initially defined for Schwartz functions $f_{j}$ on $\mathbb{R}^{n}$ admits a bounded extension

$$
T_{m}: L^{p_{1}}\left(\mathbb{R}^{n}\right) \times \cdots \times L^{p_{k}}\left(\mathbb{R}^{n}\right) \longmapsto L^{p}\left(\mathbb{R}^{n}\right) .
$$

In this case we call $m$ the symbol of $T_{m}$. We will denote by $\mathcal{M}_{p_{1}, p_{2}, \ldots, p_{k}, p}\left(\mathbb{R}^{n}\right)$ the set of all $k$-linear multipliers $m$ such that the corresponding operator $T_{m}$

Received December 13, 2004.

1991 Mathematics Subject Classification: Primary 43B20, 42B25; Secondary 46B70, 47B38.

Work of both authors is supported by the NSF. 
satisfies (1.1). The norm of $m$ in $\mathcal{M}_{p_{1}, p_{2}, \ldots, p_{k}, p}\left(\mathbb{R}^{n}\right)$ is defined as the norm of $T_{m}$.

Nontrivial examples of functions in $\mathcal{M}_{p_{1}, p_{2}, p}(\mathbb{R})$ are characteristic functions of half-planes (see [7], [8]) when $p_{1}^{-1}+p_{2}^{-1}=p^{-1}<3 / 2$ and characteristic functions of planar ellipses when $p_{1}^{-1}+p_{2}^{-1}=p^{-1}$ and $2 \leq p_{1}, p_{2}, p^{\prime}<\infty$ (see [4]). Here $p^{\prime}=p /(p-1)$. It is still an open question whether the results of this paper hold if $n=1$. In this work we show that this is not the case for the characteristic function of the ball in $\mathbb{R}^{2 n}$ if $1 / p+1 / q=1 / r$ and exactly one of $p, q$, or $r^{\prime}$ is less than 2 . We will construct a counterexample when $n=2$ and $r>2$. The general result will follow from duality and a multilinear version of de Leeuw's theorem [1].

\section{§2. Bilinearization of Fefferman's counterexample for $\mathcal{M}_{p, q, r}\left(\mathbb{R}^{2}\right)$}

For a rectangle $R$ in $\mathbb{R}^{2}$, let $R^{\prime}$ be the union of the two copies of $R$ adjacent to $R$ in the direction of its longest side. Hence, $R \cup R^{\prime}$ is a rectangle three times as long as $R$ with the same center. Key to this argument is the following geometric lemma whose proof can be found in [9], page 435 or [3], page 738 .

Lemma 1. Let $\delta>0$ be given. Then there exists a measurable subset $E$ of $\mathbb{R}^{2}$ and a finite collection of rectangles $R_{j}$ in $\mathbb{R}^{2}$ such that

(1) The $R_{j}$ are pairwise disjoint.

(2) We have $1 / 2 \leq|E| \leq 3 / 2$.

(3) We have $|E| \leq \delta \sum_{j}\left|R_{j}\right|$.

(4) For all $j$ we have $\left|R_{j}^{\prime} \cap E\right| \geq \frac{1}{12}\left|R_{j}\right|$.

Let $\delta>0$ and let $E$ and $R_{j}$ be as in Lemma 1. The proof of Lemma 1 implies that there are $2^{k}$ rectangles $R_{j}$ of dimension $2^{-k} \times 3 \log (k+2)$. Here, $k$ is chosen so that $k+2 \geq e^{1 / \delta}$. Let $v_{j}$ be the unit vector in $\mathbb{R}^{2}$ parallel to the longest side of $R_{j}$ and in the direction of the set $E$ relative to $R_{j}$.

Proposition 1. Let $R$ be a rectangle in $\mathbb{R}^{2}$ and let $v$ be a unit vector in $\mathbb{R}^{2}$ parallel to the longest side of $R$. Let $R^{\prime}$ be as above. Consider the half space $\mathcal{H}_{v}$ of $\mathbb{R}^{4}$ defined by

$$
\mathcal{H}_{v}=\left\{(\xi, \eta) \in \mathbb{R}^{2} \times \mathbb{R}^{2}:(\xi+\eta) \cdot v \geq 0\right\} .
$$


Then the following estimate is valid for all $x \in \mathbb{R}^{2}$ :

$$
\left|\int_{\mathbb{R}^{2}} \int_{\mathbb{R}^{2}} \chi_{\mathcal{H}_{v}}(\xi, \eta) \widehat{\chi R}(\xi) \widehat{\chi R}(\eta) e^{2 \pi i x \cdot(\xi+\eta)} d \xi d \eta\right| \geq \frac{1}{10} \chi_{R^{\prime}}(x) .
$$

Proof. We introduce a rotation (i.e. orthogonal matrix) $\mathcal{O}$ of $\mathbb{R}^{2}$ such that $\mathcal{O}(v)=(1,0)$. Setting $\xi=\left(\xi_{1}, \xi_{2}\right), \eta=\left(\eta_{1}, \eta_{2}\right)$ we can write the expression on the left in (2.1) as

$$
\begin{aligned}
& \left|\iint_{\mathcal{O}^{-1}(\xi+\eta) \cdot v \geq 0} \widehat{\chi_{R}}\left(\mathcal{O}^{-1} \xi\right) \widehat{\chi_{R}}\left(\mathcal{O}^{-1} \eta\right) e^{2 \pi i x \cdot \mathcal{O}^{-1}(\xi+\eta)} d \xi d \eta\right| \\
& \quad=\left|\iint_{\xi_{1}+\eta_{1} \geq 0} \widehat{\chi_{\mathcal{O}[R]}}(\xi) \widehat{\chi_{\mathcal{O}[R]}}(\eta) e^{2 \pi i \mathcal{O} x \cdot(\xi+\eta)} d \xi d \eta\right|
\end{aligned}
$$

Now the rectangle $\mathcal{O}[R]$ has sides parallel to the axes, say $\mathcal{O}[R]=I_{1} \times I_{2}$. Assume that $\left|I_{1}\right|>\left|I_{2}\right|$, i.e. its longest side is horizontal. Let $H$ be the classical Hilbert transform on the line. Setting $\mathcal{O} x=\left(y_{1}, y_{2}\right)$ we can write the last displayed expression as

$$
\begin{aligned}
& \left|\chi_{I_{2}}\left(y_{2}\right)^{2} \int_{\xi_{1} \in \mathbb{R}} \widehat{\chi_{I_{1}}}\left(\xi_{1}\right) e^{2 \pi i y_{1} \xi_{1}} \int_{\eta_{1} \geq-\xi_{1}} \widehat{\chi_{I_{1}}}\left(\eta_{1}\right) e^{2 \pi i y_{1} \eta_{1}} d \eta_{1} d \xi_{1}\right| \\
& \quad=\chi_{I_{2}}\left(y_{2}\right)\left|\int_{\xi_{1} \in \mathbb{R}} \widehat{\chi_{I_{1}}}\left(\xi_{1}\right) \frac{1}{2}(I+i H)\left[\chi_{I_{1}}(\cdot) e^{2 \pi i \xi_{1}(\cdot)}\right]\left(y_{1}\right) d \xi_{1}\right| \\
& \quad=\chi_{I_{2}}\left(y_{2}\right)\left|\frac{1}{2}(I+i H)\left(\chi_{I_{1}}\right)\left(y_{1}\right)\right|=\left|\left[\chi_{\xi_{1} \geq 0} \widehat{\chi_{I_{1} \times I_{2}}}\left(\xi_{1}, \xi_{2}\right)\right]^{\vee}\left(y_{1}, y_{2}\right)\right| .
\end{aligned}
$$

Using the result from [3] (Proposition 10.1.2) or [9] (estimate (33), page 453) we deduce that the previous expression is at least

$$
\frac{1}{10} \chi_{\left(I_{1} \times I_{2}\right)^{\prime}}\left(y_{1}, y_{2}\right)=\frac{1}{10} \chi_{(\mathcal{O}[R])^{\prime}}(\mathcal{O} x)=\frac{1}{10} \chi_{R^{\prime}}(x) .
$$

This proves the required conclusion.

Next we have the following result concerning bilinear operators on $\mathbb{R}^{2}$ of the form

$$
\begin{aligned}
T_{m}(f, g)(x)=\int_{\mathbb{R}^{2}} \int_{\mathbb{R}^{2}} m\left(\xi_{1}, \xi_{2}, \eta_{1}, \eta_{2}\right) & \widehat{f}\left(\xi_{1}, \xi_{2}\right) \widehat{g}\left(\eta_{1}, \eta_{2}\right) \\
& \times e^{2 \pi i x \cdot\left(\xi_{1}+\eta_{1}, \xi_{2}+\eta_{2}\right)} d \xi_{1} d \xi_{2} d \eta_{1} d \eta_{2} .
\end{aligned}
$$


LEMMA 2. Let $v_{1}, v_{2}, \ldots, v_{j}, \ldots$ be a sequence of unit vectors in $\mathbb{R}^{2}$. Define a sequence of half-spaces $\mathcal{H}_{v_{j}}$ in $\mathbb{R}^{4}$ as in Proposition 1 . Let $B, B^{* 1}$, $B^{* 2}$ be the following sets in $\mathbb{R}^{4}$

$$
\begin{aligned}
B & =\left\{(\xi, \eta) \in \mathbb{R}^{2} \times \mathbb{R}^{2}:|\xi|^{2}+|\eta|^{2} \leq 1\right\} \\
B^{* 1} & =\left\{(\xi, \eta) \in \mathbb{R}^{2} \times \mathbb{R}^{2}:|\xi+\eta|^{2}+|\eta|^{2} \leq 1\right\} \\
B^{* 2} & =\left\{(\xi, \eta) \in \mathbb{R}^{2} \times \mathbb{R}^{2}:|\xi|^{2}+|\xi+\eta|^{2} \leq 1\right\} .
\end{aligned}
$$

Assume that one of $T_{\chi_{B}}, T_{\chi_{B * 1}}, T_{\chi_{B^{* 2}}}$ lies in $\mathcal{M}_{p, q, r}\left(\mathbb{R}^{2}\right)$ and has norm $C=C(p, q, r)$. Then we have the following vector-valued inequality

$$
\left\|\left(\sum_{j}\left|T_{\chi_{\mathcal{H}_{j}}}\left(f_{j}, g_{j}\right)\right|^{2}\right)^{1 / 2}\right\|_{r} \leq C\left\|\left(\sum_{j}\left|f_{j}\right|^{2}\right)^{1 / 2}\right\|_{p}\left\|\left(\sum_{j}\left|g_{j}\right|^{2}\right)^{1 / 2}\right\|_{q} .
$$

for all functions $f_{j}$ and $g_{j}$.

Proof. We begin with the assumption that $T_{\chi_{B}}$ lies in $\mathcal{M}_{p, q, r}\left(\mathbb{R}^{2}\right)$ for some $p, q, r>0$. Set $\xi=\left(\xi_{1}, \xi_{2}\right)$ and $\eta=\left(\eta_{1}, \eta_{2}\right) \in \mathbb{R}^{2}$. For $\rho>0$ we define sets

$$
\begin{aligned}
B_{\rho} & =\left\{(\xi, \eta) \in \mathbb{R}^{2} \times \mathbb{R}^{2}:|\xi|^{2}+|\eta|^{2} \leq 2 \rho^{2}\right\} \\
B_{j, \rho} & =\left\{(\xi, \eta) \in \mathbb{R}^{2} \times \mathbb{R}^{2}:\left|\xi-\rho v_{j}\right|^{2}+\left|\eta-\rho v_{j}\right|^{2} \leq 2 \rho^{2}\right\} .
\end{aligned}
$$

Note that bilinear multiplier norms are translation and dilation invariant. Easy computations give that

$$
\left\|\chi_{B_{j, \rho}}\right\|_{\mathcal{M}_{p, q, r}\left(\mathbb{R}^{2}\right)} \leq\left\|\chi_{B_{\rho}}\right\|_{\mathcal{M}_{p, q, r}\left(\mathbb{R}^{2}\right)}=C .
$$

The important observation is that $\chi_{B_{j, \rho}} \rightarrow \chi_{\mathcal{H}_{v_{j}}}$ pointwise as $\rho \rightarrow \infty$ and that the multiplier norms of the functions $\chi_{B_{j, \rho}}$ are bounded above by $C$.

Moreover, by the bilinear version of a theorem of Marcinkiewicz and Zygmund ([5], Section 9), we have the following inequality for all $\rho>0$.

$$
\left\|\left(\sum_{j}\left|T_{\chi_{B_{\rho}}}\left(f_{j}, g_{j}\right)\right|^{2}\right)^{1 / 2}\right\|_{r} \leq C\left\|\left(\sum_{j}\left|f_{j}\right|^{2}\right)^{1 / 2}\right\|_{p}\left\|\left(\sum_{j}\left|g_{j}\right|^{2}\right)^{1 / 2}\right\|_{q} .
$$

Since $\chi_{B_{j, \rho}} \rightarrow \chi_{\mathcal{H}_{v_{j}}}$ pointwise as $\rho \rightarrow \infty$, we can deduce that

$$
\lim _{\rho \rightarrow \infty} T_{\chi_{B_{j, \rho}}}(f, g)(x)=T_{\chi_{\mathcal{H}_{v_{j}}}}(f, g)(x)
$$


for all $x \in \mathbb{R}^{2}$ and suitable functions $f$ and $g$. We note that the curvature of the ball $B$ is used here. By Fatou's lemma we conclude

$$
\left\|\left(\sum_{j}\left|T_{\chi_{\mathcal{H}_{v_{j}}}}\left(f_{j}, g_{j}\right)\right|^{2}\right)^{1 / 2}\right\|_{r} \leq \liminf _{\rho \rightarrow \infty}\left\|\left(\sum_{j}\left|T_{\chi_{B_{j, \rho}}}\left(f_{j}, g_{j}\right)\right|^{2}\right)^{1 / 2}\right\|_{r}
$$

Now, observe the following identity:

$$
T_{\chi_{B_{j, \rho}}}(f, g)(x)=e^{4 \pi i \rho v_{j} \cdot x} T_{\chi_{B_{\rho}}}\left(e^{-2 \pi i \rho v_{j} \cdot(\cdot)} f, e^{-2 \pi i \rho v_{j} \cdot(\cdot)} g\right)(x) .
$$

Using (2.2) and the previous identity gives

$$
\begin{aligned}
& \left\|\left(\sum_{j}\left|T_{\chi_{\mathcal{H}_{j}}}\left(f_{j}, g_{j}\right)\right|^{2}\right)^{1 / 2}\right\|_{r} \\
& \leq \liminf _{\rho \rightarrow \infty}\left\|\left(\sum_{j}\left|e^{4 \pi i \rho v_{j} \cdot(\cdot)} T_{\chi_{B_{\rho}}}\left(e^{-2 \pi i \rho v_{j} \cdot(\cdot)} f_{j}, e^{-2 \pi i \rho v_{j} \cdot(\cdot)} g_{j}\right)\right|^{2}\right)^{1 / 2}\right\|_{r} \\
& \leq \liminf _{\rho \rightarrow \infty}\left\|\chi_{B_{\rho}}\right\|_{\mathcal{M}_{p, q, r}} \\
& \quad \times\left\|\left(\sum_{j}\left|e^{-2 \pi i \rho v_{j} \cdot(\cdot)} f_{j}\right|^{2}\right)^{1 / 2}\right\|_{p}\left\|\left(\sum_{j}\left|e^{-2 \pi i \rho v_{j} \cdot(\cdot)} g_{j}\right|^{2}\right)^{1 / 2}\right\|_{q} \\
& =C\left\|\left(\sum_{j}\left|f_{j}\right|^{2}\right)^{1 / 2}\right\|\left\|_{p}\right\|\left(\sum_{j}\left|g_{j}\right|^{2}\right)^{1 / 2} \|_{q}
\end{aligned}
$$

where the last equality follows from the dilation invariance of bilinear multiplier norms.

The proof of the analogous statements for $T_{B^{* 1}}$ and $T_{B^{* 2}}$ is as follows. We introduce sets

$$
\begin{aligned}
& B_{\rho}^{* 1}=\left\{(\xi, \eta) \in \mathbb{R}^{2} \times \mathbb{R}^{2}:|\xi+\eta|^{2}+|\eta|^{2} \leq \rho^{2}\right\} \\
& B_{j, \rho}^{* 1}=\left\{(\xi, \eta) \in \mathbb{R}^{2} \times \mathbb{R}^{2}:\left|\xi-\rho v_{j}+\eta\right|^{2}+|\eta|^{2} \leq \rho^{2}\right\} \\
& B_{\rho}^{* 2}=\left\{(\xi, \eta) \in \mathbb{R}^{2} \times \mathbb{R}^{2}:|\xi|^{2}+|\xi+\eta|^{2} \leq \rho^{2}\right\} \\
& B_{j, \rho}^{* 2}=\left\{(\xi, \eta) \in \mathbb{R}^{2} \times \mathbb{R}^{2}:|\xi|^{2}+\left|\xi+\eta-\rho v_{j}\right|^{2} \leq \rho^{2}\right\} .
\end{aligned}
$$

Note that both $B_{j, \rho}^{* 1}$ and $B_{j, \rho}^{* 2}$ converge to $\mathcal{H}_{v_{j}}$ as $\rho \rightarrow \infty$. Using the identities

$$
\begin{aligned}
& T_{\chi_{B_{j, \rho}^{* 1}}}(f, g)(x)=e^{2 \pi i \rho v_{j} \cdot x} T_{\chi_{B_{\rho}^{* 1}}}\left(e^{-2 \pi i \rho v_{j} \cdot(\cdot)} f, g\right)(x) \\
& T_{\chi_{B_{j, \rho}^{* 2}}}(f, g)(x)=e^{2 \pi i \rho v_{j} \cdot x} T_{\chi_{B_{\rho}^{* 2}}}\left(f, e^{-2 \pi i \rho v_{j} \cdot(\cdot)} g\right)(x),
\end{aligned}
$$

we obtain a similar conclusion for the bilinear operators $T_{\chi_{B^{* 1}}}$ and $T_{\chi_{B^{* 2}}}$. 
The next ingredient that we will need is a multilinear version of de Leeuw's theorem. For $1 \leq j \leq k$ we will consider $\xi_{j} \in \mathbb{R}^{n}, \eta_{j} \in \mathbb{R}^{m}$. Then the pairs $\left(\xi_{j}, \eta_{j}\right) \in \mathbb{R}^{n+m}$. Also for a function $f$ on $\mathbb{R}^{n}$ and $g$ on $\mathbb{R}^{m}$ we introduce another function $f \otimes g$ on $\mathbb{R}^{n+m}$ by setting $(f \otimes g)(\xi, \eta)=f(\xi) g(\eta)$.

Proposition 2. Suppose that

$$
m\left(\xi_{1}, \eta_{1}, \xi_{2}, \eta_{2}, \ldots, \xi_{k}, \eta_{k}\right) \in \mathcal{M}_{p_{1}, p_{2}, \ldots, p_{k}, p}\left(\mathbb{R}^{n+m}\right)
$$

for some $1<p<\infty$. Then for almost every $\left(\xi_{1}, \ldots, \xi_{k}\right) \in\left(\mathbb{R}^{n}\right)^{k}$ the function $m\left(\xi_{1}, \cdot, \xi_{2}, \cdot, \ldots, \xi_{k}, \cdot\right)$ lies in $\mathcal{M}_{p_{1}, p_{2}, \ldots, p_{k}, p}\left(\mathbb{R}^{m}\right)$, with norm

$$
\left\|m\left(\xi_{1}, \cdot, \xi_{2}, \cdot, \ldots, \xi_{k}, \cdot\right)\right\|_{\mathcal{M}_{p_{1}, p_{2}, \ldots, p_{k}, p}\left(\mathbb{R}^{m}\right)} \leq\|m\|_{\mathcal{M}_{p_{1}, p_{2}, \ldots, p_{k}, p}\left(\mathbb{R}^{n+m}\right)} .
$$

Proof. In the proof that follows for simplicity we take $k=2$. The case of a general $k$ does not present any complications, only notational changes. We also assume that $m$ is continuous. This assumption may be easily removed by considering convolutions of $m$ in each variable with smooth approximate identities.

Fix $f_{1}, g_{1}, h_{1} \in \mathcal{S}\left(\mathbb{R}^{n}\right)$ and $f_{2}, g_{2}, h_{2} \in \mathcal{S}\left(\mathbb{R}^{m}\right)$ with $\left\|f_{2}\right\|_{p_{1}}=\left\|g_{2}\right\|_{p_{2}}=$ $\left\|h_{2}\right\|_{p^{\prime}}=1$. Let

$$
\begin{aligned}
M\left(\xi_{1}, \xi_{2}\right)=\int_{\mathbb{R}^{m}} \int_{\mathbb{R}^{m}} \int_{\mathbb{R}^{m}} m\left(\xi_{1}, \eta_{1}, \xi_{2}, \eta_{2}\right) \widehat{f_{2}}\left(\eta_{1}\right) \widehat{g_{2}}\left(\eta_{2}\right) \\
\times e^{2 \pi i\left(\eta_{1}+\eta_{2}\right) \cdot x_{2}} d \eta_{1} d \eta_{2} h_{2}\left(x_{2}\right) d x_{2} .
\end{aligned}
$$

If we can show that $M \in \mathcal{M}_{p_{1}, p_{2}, p}\left(\mathbb{R}^{n}\right)$, then by Proposition 4 (vi) in [6], we can deduce that $\|M\|_{\infty} \leq\|M\|_{\mathcal{M}_{p_{1}, p_{2}, p}}$. Then, by duality, it will follow that $\left\|T_{m}\left(f_{2}, g_{2}\right)\right\|_{p} \leq\|M\|_{\infty} \leq\|M\|_{\mathcal{M}_{p_{1}, p_{2}, p}}$. We have

$$
\begin{aligned}
\left|\left\langle T_{M}\left(f_{1}, g_{1}\right), h_{1}\right\rangle\right| & \\
= & \int_{\mathbb{R}^{n}} \int_{\mathbb{R}^{n}} \int_{\mathbb{R}^{n}} M\left(\xi_{1}, \xi_{2}\right) \widehat{f_{1}}\left(\xi_{1}\right) \widehat{g_{1}}\left(\xi_{2}\right) e^{2 \pi i\left(\xi_{1}+\xi_{2}\right) \cdot x_{1}} d \xi_{1} d \xi_{2} h_{1}\left(x_{1}\right) d x_{1} \\
= & \int_{\mathbb{R}^{n}} \int_{\mathbb{R}^{n}} \int_{\mathbb{R}^{n}} \int_{\mathbb{R}^{m}} \int_{\mathbb{R}^{m}} \int_{\mathbb{R}^{m}} m\left(\xi_{1}, \eta_{1}, \xi_{2}, \eta_{2}\right) \widehat{f_{2}}\left(\eta_{1}\right) \widehat{g_{2}}\left(\eta_{2}\right) e^{2 \pi i\left(\eta_{1}+\eta_{2}\right) \cdot x_{2}} \\
= & \int_{\mathbb{R}^{n+m}} \int_{\mathbb{R}^{n+m}} \int_{\mathbb{R}^{n+m}} m\left(\xi_{1}, \eta_{1}, \xi_{2}, \eta_{2}\right) \widehat{f}_{1}\left(\xi_{1}\right) \widehat{f_{2}}\left(\eta_{1}\right) \widehat{g_{1}}\left(\xi_{2}\right) \widehat{g_{2}}\left(\eta_{2}\right) \\
\quad & \times e^{2 \pi i\left(\left(\xi_{1}, \eta_{1}\right)+\left(\xi_{2}, \eta_{2}\right)\right) \cdot\left(x_{1}, x_{2}\right)} d\left(\xi_{1}, \eta_{1}\right) d\left(\xi_{2}, \eta_{2}\right) h_{1}\left(x_{1}\right) h_{2}\left(x_{2}\right) d\left(x_{1}, x_{2}\right)
\end{aligned}
$$




$$
\begin{aligned}
& =\left|\left\langle T_{m}\left(f_{1} \otimes f_{2}, g_{1} \otimes g_{2}\right), h_{1} \otimes h_{2}\right\rangle\right| \\
& \leq\|m\|_{\mathcal{M}_{p_{1}, p_{2}, p}\left(\mathbb{R}^{n+m}\right)}\left\|f_{1} \otimes f_{2}\right\|_{p_{1}}\left\|g_{1} \otimes g_{2}\right\|_{p_{2}}\left\|h_{1} \otimes h_{2}\right\|_{p^{\prime}} \\
& =\|m\|_{\mathcal{M}_{p_{1}, p_{2}, p}\left(\mathbb{R}^{n+m}\right)}\left\|f_{1}\right\|_{p_{1}}\left\|f_{2}\right\|_{p_{1}}\left\|g_{1}\right\|_{p_{2}}\left\|g_{2}\right\|_{p_{2}}\left\|h_{1}\right\|_{p}\left\|h_{2}\right\|_{p^{\prime}} \\
& =\|m\|_{\mathcal{M}_{p_{1}, p_{2}, p}\left(\mathbb{R}^{n+m}\right)}\left\|f_{1}\right\|_{p_{1}}\left\|g_{1}\right\|_{p_{2}}\left\|h_{1}\right\|_{p^{\prime}},
\end{aligned}
$$

where the inequality follows from the boundedness of $T_{m}$.

The following is the main result of this article.

Theorem 1. Let $n>1$ and $1 / p+1 / q=1 / r$ with exactly one of $p, q$, or $r^{\prime}$ less than 2 . Let $B$ be the unit ball in $\mathbb{R}^{2 n}$. Then $\chi_{B} \notin \mathcal{M}_{p, q, r}\left(\mathbb{R}^{n}\right)$.

Proof. Using Proposition 2 and considering the two dual operators $T_{\chi_{B^{* 1}}}$ and $T_{\chi_{B^{* 2}}}$ of $T_{\chi_{B}}$, it suffices to show that all of these operators are not in $\mathcal{M}_{p, q, r}\left(\mathbb{R}^{2}\right)$ for $p, q, r>2$. Therefore, we fix $n=2$ and $p, q, r$ satisfying $p^{-1}+q^{-1}=r^{-1}<1 / 2$. We suppose that $\chi_{B}$ is in $\mathcal{M}_{p, q, r}\left(\mathbb{R}^{2}\right)$ with norm $C$.

Suppose that $\delta>0$ is given. Let $E$ and $R_{j}$ be as in Lemma 1 . Let $v_{j}$ be the the unit vector parallel to the longest side of $R_{j}$ and pointing in the direction of the set $E$ relative to $R_{j}$. In the spirit of Fefferman's argument, we estimate $\sum_{j} \int_{E}\left|T_{j}\left(\chi_{R_{j}}, \chi_{R_{j}}\right)(x)\right|^{2} d x$ from above and below and arrive to a contradiction. We have

$$
\begin{aligned}
& \sum_{j} \int_{E}\left|T_{\mathcal{H}_{v_{j}}}\left(\chi_{R_{j}}, \chi_{R_{j}}\right)(x)\right|^{2} d x \\
& \leq|E|^{\frac{r-2}{r}}\left\|\left(\sum_{j}\left|T_{\mathcal{H}_{v_{j}}}\left(\chi_{R_{j}}, \chi_{R_{j}}\right)\right|^{2}\right)^{1 / 2}\right\|_{r}^{2} \\
& \leq C|E|^{\frac{r-2}{r}}\left\|\left(\sum_{j}\left|\chi_{R_{j}}\right|^{2}\right)^{1 / 2}\right\|_{p}^{2}\left\|\left(\sum_{j}\left|\chi_{R_{j}}\right|^{2}\right)^{1 / 2}\right\|_{q}^{2} \quad \text { (bölder's Lemma 2) } \\
& =C|E|^{\frac{r-2}{r}}\left(\sum_{j}\left|R_{j}\right|\right)^{2 / r} \quad\left(\text { by the disjointness of the } R_{j} \mathrm{~s}\right) \\
& \leq C \delta^{\frac{r-2}{r}} \sum_{j}\left|R_{j}\right| \quad(\text { Lemma } 1) .
\end{aligned}
$$


For the reverse inequality we argue as follows:

$$
\begin{aligned}
& \sum_{j} \int_{E}\left|T_{\mathcal{H}_{v_{j}}}\left(\chi_{R_{j}}, \chi_{R_{j}}\right)(x)\right|^{2} d x \\
& \geq \sum_{j} \int_{E}\left(\frac{1}{10} \chi_{R_{j}^{\prime}}(x)\right)^{2} d x \quad \text { (Proposition 1) } \\
& \quad=\frac{1}{100} \sum_{j}\left|E \cap R_{j}^{\prime}\right| \\
& \geq \frac{1}{1200} \sum_{j}\left|R_{j}\right| \quad \text { (Lemma 1). }
\end{aligned}
$$

Putting these two estimates together, we obtain that

$$
\frac{1}{1200} \sum_{j}\left|R_{j}\right| \leq C \delta^{\frac{r-2}{r}} \sum_{j}\left|R_{j}\right|
$$

and therefore

$$
\frac{1}{1200} \leq C \delta^{\frac{r-2}{r}}
$$

for any $\delta>0$. This is a contradiction since $r>2$.

The authors would like to thank Maria Carmen Reguera-Rodríguez for pointing out an oversight in an earlier version of this manuscript.

\section{REFERENCES}

[1] K. de Leeuw, On $L_{p}$ multipliers, Ann. of Math., 81 (1965), 364-379.

[2] C. Fefferman, The multiplier problem for the ball, Ann. of Math., 94 (1971), 330-336.

[3] L. Grafakos, Classical and Modern Fourier Analysis, Prentice Hall, Upper Saddle River, NJ, 2003.

[4] L. Grafakos and X. Li, The disc as a bilinear multiplier, Amer. J. Math., 128 (2006), 91-119.

[5] L. Grafakos and J. M. Martell, Extrapolation of weighted norm inequalities for multivariable operators, J. of Geom. Anal., 14 (2004), 19-46.

[6] L. Grafakos and R. Torres, Multilinear Calderoón-Zygmund theory, Adv. in Math. J., 165 (2002), 124-164.

[7] M. T. Lacey and C. M. Thiele, $L^{p}$ bounds for the bilinear Hilbert transform, $p>2$, Ann. of Math., 146 (1997), 693-724.

[8] M. Lacey and C. Thiele, On Calderón's conjecture, Ann. of Math., 149 (1999), 475496.

[9] E. M. Stein, Harmonic Analysis, Real Variable Methods, Orthogonality, and Oscillatory Integrals, Princeton Univ. Press, Princeton, NJ, 1993. 
Geoff Diestel

Department of Mathematics

University of South Carolina

Columbia, SC 29208

$U S A$

diestelg@math.sc.edu

Loukas Grafakos

Department of Mathematics

University of Missouri

Columbia, MO 65211

USA

loukas@math.missouri.edu 